\title{
Leiomyoma as a cause of urinary retention
}

\section{Kemal Sarsmaz, Asli Goker, Naci Kemal Kuscu \\ Department of Obstetrics and Gynecology, University of Celal Bayar, Turkey}

\section{Abstract}

Urinary retention is uncommon in reproductive age women. The frequent causes for obstructive retention are pelvic masses and a history of surgery. We report a case of intermittent urinary retention caused by a leiomyoma. A 47-year old patient with voiding difficulties for the past four months was referred to the gynecology outpatient clinic. She had urinary retention and had been catheterized several times for bladder emptying. She had been evaluated by the urology clinic and a cystoscopy was performed with normal findings. Magnetic resonance imaging (MRI) revealed a pelvic mass at the anterior uterine wall with a diameter of 10 $\mathrm{cm}$. Her physical examination confirmed the MRI findings. Total abdominal hysterectomy was performed with a complete resolution of the patient's complaints. Women with urinary retention should be consultated with a gynecologist in order to rule out pelvic masses.

\section{Introduction}

Acute urinary retention is defined as the inability of voluntary voiding resulting in bladder over-distention usually requiring catheterization for pain relief. Infection and renal failure are important complications if left untreated. The incidence in women is not well documented. Causes for urinary retention are classified as obstructive, infectious and inflammatory, pharmacologic, neurologic, or other. Obstruction may occur intrinsically as a bladder stone or stricture and extrinsically as a compression caused by a mass. The gynecologist encounters urinary retention usually in the post-surgical patient. Obstruction of the urinary path may be due to uterine prolapse or a pelvic mass causing compression. Uterine leiomyomas have been reported as a rare cause for urinary retention, however intermittent cases are even rarer. ${ }^{1}$ The obstruction of the bladder neck or urethra may cause difficulties in voiding.

\section{Case Report}

We present a 47-year old multiparous woman who was referred to our gynecology outpatient clinic with urinary difficulties. She had a history of several bladder catheterizations. Four months ago she had applied to the urology clinic because of macroscopic hematuria and could only void with a Valsalva maneuver with abdominal pain and pelvic pressure. A cystoscopy was performed showing partial collapse of the posterior bladder wall. Magnetic resonance imaging identified a $10 \times 9$ $\mathrm{cm}$ sized, single, well circumscribed leiomyoma compressing the uterus into the base of the pelvis with no signs of invasion, hence she was referred to the gynecology clinic.

Gynecologic examination revealed a 16week sized uterus. Urine examination was nondiagnostic. Transvaginal ultrasonography showed a fibroid arising from the anterior uterine wall with a diameter of $10 \mathrm{~cm}$ and bilaterally normal adnexa, slight thickening of the bladder wall. She had no history of diabetes, neurologic dysfunction or surgery. Her menstrual periods were regular. A total abdominal hysterectomy and bilateral salpingoopherectomy was performed. A large leiomyoma between the promontorium and pubis was observed pushing the uterus in a retrovert position. The patient was able to void spontaneously on the first postoperative day. She was discharged with no complaint on the third postoperative day. At 6 -week follow-up the patient had no difficulty in voiding, had no pain or pressure feeling. Postvoid bladder residuals were less than $50 \mathrm{cc}$.

\section{Discussion}

Urinary retention is rarely caused by an obstruction in reproductive aged women without a history of surgery. Pregnancy, pelvic trauma resulting in hematoma or edema and pelvic masses may cause obstruction. Most of the case reports of urinary retention are secondary to pelvic masses with acute retention. ${ }^{1}$ Yang et $a l$. presented a report of six cases where the women were able to void in a standing position only. ${ }^{2}$ Barnacle et al. reported two cases of intermittant urinary retention due to uterine leiomyomas. ${ }^{2}$ Our case is another example to the rare entity of intermittant urinary retention caused by a leiomyoma. Strong Valsalva maneuver enabled the patient to micturate with a result of abdominal pain.

The initial evaluation of a patient with void-
Correspondence: Asli Goker, Department of Obstetrics and Gynecology, Faculty of Medicine, Celal Bayar University, Manisa, Turkey.

Tel. +90.532 .471 .31 .96 - Fax: +90.236 .233 .80 .40 .

E-mail: asligoker@gmail.com

Key words: leiomyoma, urinary retention.

Contributions: the authors contributed equally.

Conflict of interests: the authors declare no potential conflict of interests.

Conference presentation: part of this paper was presented at the 5th International Urogynecology Congress 2013 April 6-7, Izmir, Turkey.

Received for publication: 28 May 2013.

Revision received: 22 October 203

Accepted for publication: 31 October 2013.

This work is licensed under a Creative Commons Attribution NonCommercial 3.0 License (CC BYNC 3.0).

(C) Copyright K. Sarsmaz et al., 2013

Licensee PAGEPress, Italy

Urogynaecologia 2013; 27:e4

doi:10.4081/uij.2013.e4

ing difficulties comprises urodynamic tests to help determine whether the woman has an obstruction or a detrusor muscle dysfunction. Another diagnostic tool is cystoscopy, however this is an invasive method and helps to diagnose lesions of the bladder only. Whenever suspected, a transvaginal ultrasound is indicated to confirm a mass compressing the bladder. Transvaginal ultrasonography gives better results about the genitourinary tract and pelvic masses $^{2}$ while better image quality is achieved by magnetic resonance imaging (MRI) to identify urethral masses or diverticula. ${ }^{3}$ The treatment of acute urinary retention is catheterization but a differential diagnosis must be made for definitive treatment.

An impacted pelvic mass may compress the proximal urethra and bladder neck leading to bladder over distention, reposition of the uterus in a retrovert position and displacing the cervix..$^{2,4}$ Another theory about pelvic mass causing urinary retention is the vascular steal effect where a blood shunt from the urogenital system results in detrusor hypotonia. ${ }^{5}$ Over distention of the bladder may cause ischemia of the detrusor and subsequent urinary tract infections. ${ }^{4}$ Pregnancy is another reason for retroversion of the uterus and urinary retention may occur as often as 1 per 3000 pregnancies. ${ }^{2}$ Yang et al. have reported several recom- 


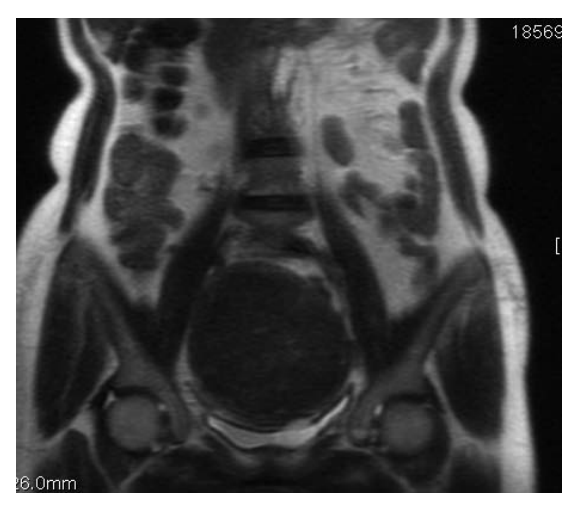

Figure 1. Sagittal magnetic resonance imaging confirmed the large subserosal leiomyoma compressing the bladder and pushing down the uterus and giving it a retroverted position.

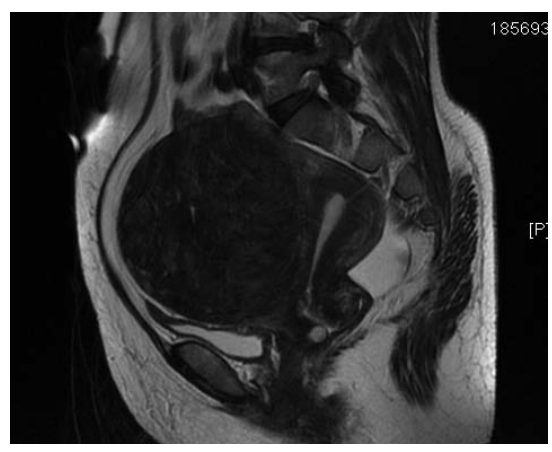

Figure 2. Coronal magnetic resonance imaging demonstrating that fibroid is filling the whole pelvic space. mendations to avoid acute retention, such as limited fluid intake before sleep, leaning forward to initiate voiding, avoiding Valsalva maneuver and using the Crede maneuver to initiate and maintain voiding. ${ }^{2}$ In case of neurologic bladder, self-catheterization may be applied. When the underlying cause is a pelvic mass, hysterectomy is the first line therapy although myomectomy, uterine artery embolization and gonadotropin releasing hormone agonist therapy have been used as well. ${ }^{4}$ The reported patient was 47 years old with no further fertility plans and the leiomyoma was $10 \mathrm{~cm}$ in diameter, therefore a hysterectomy was the choice of treatment. Conservative methods were not preferred by the patient.

In conclusion the goal of this report is to reduce the time from the first presentation to definitive treatment by reminding that pelvic masses may be the main cause of urinary retention. All women who apply with urinary problems should be evaluated by a gynecologist to rule out pelvic masses. Radiologic confirmation is helpful and both ultrasonography and MRI may be used for this purpose; however physical examination and pelvic examination are still the most important first step diagnostic methods. A complete pelvic examination is mandatory for differential diagnosis. There are alternative options for uterine leiomyoma as a cause of urinary retention but when appropriate, hysterectomy is the first line treatment choice. As a summary, urinary retention should be evaluated by urologists and gynecologists together and pelvic masses should be kept in mind.

\section{References}

1. Barnacle S, Muir T. Intermittent urinary retention secondary to a uterine leiomyoma. Int Urogynecol J 2007;18:339-41.

2. Yang JM, Huang We. Sonographic findings of acute urinary retention secondary to an impacted pelvic mass. J Ultrasound Med 2002;21:1165-9.

3. Bruschini H, Truzzi JC, Simonetti R, et al. Paraurethral leiomyoma in a female causing urinary obstruction. Int Urogynecol J 2006;17:286-9.

4. Chu MC, Dabney L, Hardart A. Treatment of urinary retention caused by uterine leiomyoma with a gonadotropin-releasing hormone agonist: case report and review. Female Pelvic Med Reconstr Surg 2013; 19:52Y55.

5. Yazdany T, Bhatia NN, Nguyen JN. Urinary retention and voiding dysfunction in women with uterine leiomyoma. J Reprod Med 2012;57:384-9. 